\title{
Knowledge Representation by Analogy for the Design of Learning and Assessment Strategies
}

\author{
Walid Mestadi \\ Ibn Tofail University, Faculty of Science, Kenitra, Morocco \\ Email: mestadi.walid@gmail.com
}

\author{
Khalid Nafil \\ Software Project Management Research Team, University Mohammed V, ENSIAS, Rabat, Morocco \\ Email: k.nafil@um5s.net.ma
}

Raja Touahni

Ibn Tofail University, Faculty of Science, Kenitra, Morocco

Email: rtouahni@hotmail.com

Rochdi Messoussi

Ibn Tofail University, Faculty of Science, Kenitra, Morocco

Email: messoussi@gmail.com

\begin{abstract}
The difficulty of learning a novel knowledge may not be the same for each learner. Often instructors try to find the easiest way to make a new knowledge understandable by most learners, but few of them give importance to the use of analogies which is the common practice in real life situations. This paper attempts to highlight that learning by analogy allows making the understanding of a novel and complicated knowledge easier. We first propose a model that represents the desired knowledge in an abstract way which allows us to find analogies in different domains. Based on these analogies, learning and assessment strategies can be derived in order to improve the learning outcome. Secondly, the design of a computer system that integrates the analogies, learning and assessment strategies into a digital learning environment is proposed.
\end{abstract}

Index Terms-Learning by Analogy, Analogy Model Design, Learning and Assessment Strategies, Knowledge Structuring, Digital Learning Systems.

\section{INTRODUCTION}

Due to the progress of information and communication technologies (ICT), access to information has become easy and fast. Nowadays, we are witnessing an exponential sharing of knowledge in all fields through digital resources (website, files, tutorial, courses and videos). Education takes advantage of the availability of these resources to improve the learning outcome. However, the variety and the amount of these resources are not sufficient to improve learning. A meaningful learning occurs when learners are able to transfer the acquired knowledge into novel situations $[15,10,14]$.
The desired knowledge contained in these digital resources should be modeled in order to make them memorable and understandable. Authors of these resources such as instructors and experts in the educational field try to represent complex (abstract or novel) knowledge either by a simple (concrete) representations $[14,12]$ and/or by using analog representations $[16,18]$. An example of this latter could be a human heart which can be represented as a pump or an electronics capacitor, an eye by a camera, a brain by a computer [6]. Also, a network routing can be represented as a road network.

Learning through analogies is a powerful mechanism $[3,1]$, specific to the human reasoning $[5,17]$ and promotes the learning outcome and the knowledge transfer from a familiar situation to a novel one $[13,2,8$, $7,6]$.

The analogy is often used in problem-solving, explanation, argumentation, inference [17], transfer of expertise (software engineering) and discovery [8]. It involves several fields of research, such as psychology, artificial intelligence, philosophy [5], medicine, economics, and law [17].

However, the use of analogies in the learning process is not obvious $[9,3]$ and can lead to misinterpretations (if it is not used carefully), especially when learners are unable to separate the acquired knowledge from the analogies $[8,13,3]$. Therefore among the issues that can be faced, we have:

- The difficulty of finding analogies that represent the desired knowledge.

- The need for a standard that describes how to integrate analogies into the learning process.

- The assurance that learners can separate the knowledge to understand from the analogy. 
- The assurance that learners can use the acquired knowledge in a novel situation.

This paper is inspired by the work of [11] which proposes a knowledge structuring for learning by levels. This paper attempts to address the issues listed above, and its main contributions can be summarized as follows:

- We propose a model and a process to support the instructor in designing analogies.

- Based on the analogies we develop learning strategies to improve learning outcome and assessment strategies to check if the learners can transfer the acquired knowledge.

- We propose a design of a computer system that aims to integrate analogies, learning and assessment strategies into a digital learning environment.

The rest of the paper is organized as follows: the next section, examines some related works, section III describes the proposed model of analogy design, section IV provides a case study illustrating the different aspects of the proposed model, section $\mathrm{V}$ describes the computer system design and finally section VI concludes the paper.

\section{STATE OF THE ART}

Several research works attempt to normalize the use of analogies in the learning process. In the design of analogies, two domains $\mathrm{A}$ and $\mathrm{B}$ are called analogs, if there is a structural matching between elements of the domain A called source and those of the domain B called target, which implies that the conclusions made in one of the domains are also true for the other $[17,4,1]$.

Duit in [1] proposes a formal definition of analogy relations; $R_{1}$ and $R_{2}$ are analogous, if they have partially or totally identical characteristics in their structures (see Fig. 1). However, the analogy relation between $R_{1}$ and $R_{2}$ represents an analogy of first level (Fig. 1 label (A)). The identical characteristics are represented by the model $R_{m}$. This latter one can be analog with another model $R_{x}$. However, the analogy relation between $R_{m}$ and $R x$ represents an analogy in a more abstract level than that between $R_{1}$ and $R_{2}$.

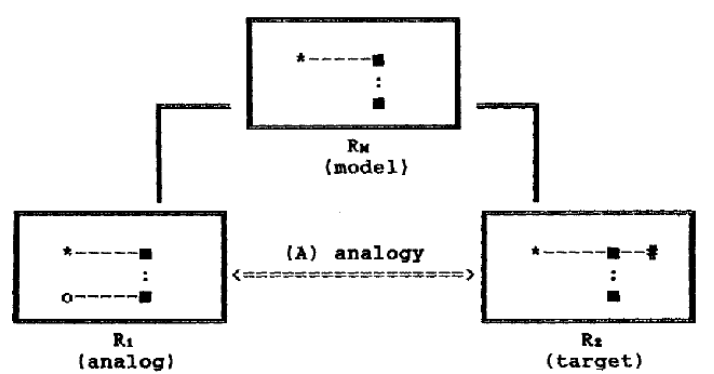

Fig.1. Comparisons of structures between two domains (extracted from [1]).

Winston in [17], proposes an object-oriented descriptive representation for the design of analogies. He focuses on the identification of the similarities between parts (desired knowledge). He combines the instances of the elements that constitute an act, such as objects, object attributes, relations, agents and additional information.

Gentner in [4], proposes a structure mapping theory. It considers the field of study as a system of objects composed of a set of attributes and linked by relations. The matching is done through four types of similarities literal, relations, object attributes and simple. These types make it possible to structure several situations when designing analogies [1].

Williams in [16], propose a design of a virtual learning game that uses analogies, in which the learner/player is asked to complete a task such as comparing between real cases and analogies cases presented side-by-side in a game world.

Mestadi in [11] propose three levels for structuring knowledge. These levels result from the observations on the use of examples for explanation. They highlighted that while switching between examples some elements may or may not change their nature. Nature here means that an element can be a physical or a logical object, an action or an agent.

\section{A. Synthesis}

The elements used in the design of analogies, namely: objects, object attributes, and object relationships, are almost similar to elements used in the object-oriented programming (OOP) paradigm. These elements are used to design the static and the dynamic aspects of information systems (IS).

The static aspect is used to design a conceptual model using instances of the elements of the OOP, for example, objects (car and person) may be linked with the relation (drive or repair). To design a robust model, it is necessary to use instances of objects in a higher level of abstraction, for example instead of using a "car" we could use a "vehicle".

The dynamic aspect is used to design conceptual model that show the transition of state and the evolution of objects in time. For example, the object instance "car" may be in one of the states: off, on, broken down or out of service and the object instance "water" may be in the liquid, solid or vapor state.

The works of $[17,4,1]$ give less importance to the dynamic aspect of the design of analogies and use elements instances in a low-level abstraction, while [16] represent the desired knowledge and the analogies only by the static aspect.

\section{The Proposed MOdEL}

In this section, the mechanisms behind the transition from a standard representation of knowledge into one or more analogies representations are described.

In a learning session, often, the instructor gives analogies between elements that may not have the same structure and nature. Generally, the element in one field does not have the same nature as those in another one, for instance, "human brain" could be compared to a 
"Computer Microprocessor", this similarity could be represented in a static and/or in a dynamic aspect.

The abstract representation of elements of a field of study is important in finding analog examples. The abstraction could be defined as the isolation of the elements from their context (avoiding the specific characteristics of the field), and their projection in elements of another field that may have or not the same nature [11]. Therefore, the nature of the elements of a field of study can be:

- A physical agent (i.e. a living being)

- A logical agent (i.e. artificial intelligence)

- A physical object (i.e. a machine, table)

- A logical object (i.e. a computer program)

- A physical action (i.e. controlling, hitting, producing, consuming)

- A logical action (i.e. thinking, talking)

Table 1 illustrates an example of the representation of the elements with and without respecting their nature.

Table 1. Two examples of elements representation with and without respecting their nature.

\begin{tabular}{|l|l|}
\hline \multicolumn{2}{|c|}{ Element of the domain } \\
\hline $\begin{array}{l}\text { [person] [control or drive] } \\
\text { [car] }\end{array}$ & [secretary] [treat] [text] \\
\hline \multicolumn{2}{|c|}{ With nature } \\
\hline $\begin{array}{l}\text { [physical agent] [physical } \\
\text { action name: control or drive] } \\
\text { [physical object] }\end{array}$ & $\begin{array}{l}\text { [physical agent] [physical action } \\
\text { name: treat or change] [logical } \\
\text { object] }\end{array}$ \\
\hline \multicolumn{2}{|c|}{ Without nature } \\
\hline $\begin{array}{l}\text { [object] [action name: } \\
\text { control or drive] [object] }\end{array}$ & $\begin{array}{l}\text { [object] [action name: treat or } \\
\text { change] [object] }\end{array}$ \\
\hline
\end{tabular}

Representing an element with another having the same nature imposes a strong constraint when searching of analogies. If an element's nature is "physical or logical object", the correspondent element must have the same nature, on the other hand representing an element without taking into consideration its nature does not make a constraint for the correspondent element, since it is represented as "object".

The proposed process of the design of the analogies is shown in Fig. 2.

The process consists of the following steps:

- Static aspect representation: in which the main elements of the field are identified. Also, the functions of each one and the relations between them at a higher abstraction level are identified.

- Dynamic aspect representation: in which the interactions of theses elements with or without respecting their nature are defined.

- Analogies: in which we search instances that respect the interactions of the elements with or without respecting the nature.

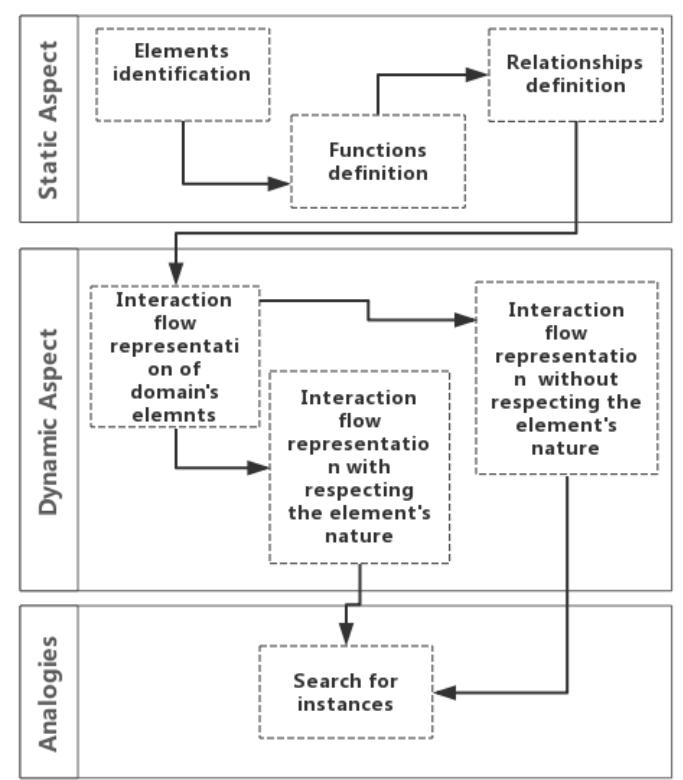

Fig.2. The proposed process for the design of the analogies

The design of the dynamic aspect allows designing the behavior of elements in response to actions made by other elements.

\section{CASE Study}

In this section, we present our case study that shows the use of the proposed model to elaborate analogies which allow us to design learning and assessment strategies that could be integrated into the learning process.

Let's assume that the desired knowledge is from the field of medicine, specifically the Blood Circulation also called Circulatory System (CS). Intuitively, an analogy can be identified, such as the Wastewater treatment (WT). The elements that make up a WT have several characteristics in common with those of the CS, are the pumping, the canalization, the treatment, etc. The study of the WT can be a good learning ground to understand the concept of the CS.

\section{A. Static aspect representation}

To represent the static aspect, we first identify the elements, their functions and the relations between them (Table 2). Therefore, the main elements involved in the CS are: "heart", "Lungs", "Organs" and "Blood".

Table 2. Functions performed by each element using infinitive verbs and their synonyms in a high level of abstraction

\begin{tabular}{|l|l|l|l|}
\hline Heart & Lungs & Organs & Blood \\
\hline $\begin{array}{l}\text { Amplifies, feeds } \\
\text { or pumps }\end{array}$ & Treat & Treat \\
& $\begin{array}{l}\text { positively, } \\
\text { enriches or } \\
\text { recycles }\end{array}$ & $\begin{array}{l}\text { Transports } \\
\text { consume or } \\
\text { deplete }\end{array}$ & $\begin{array}{l}\text { or } \\
\text { circulates }\end{array}$ \\
\hline
\end{tabular}

The verbs and their synonyms are used to identify other elements who share the same functions, such as, the function pumping of the element «heart» which could be founded in a capacitor, a water pump...etc. 
The definition of relations between the elements such as sending and receiving the blood between the heart, the lungs and the organs, allows us to refine the search of correspondent elements. The static aspect alone does not allow the identification of concepts hardly perceptible such as the cycle concept which is fundamental in the CS. Therefore the dynamic representation is important.

\section{B. Dynamic aspect representation}

The dynamic aspect consists of the representation of flow interaction between elements of study's field, then an abstract representation of those elements while respecting their nature and finally, an abstract representation without respecting the nature of those elements. The table 3 below gives an example of these three representations. The representation of the interaction flow by a descriptive form may cause a difficulty in the interpretation (Table 3). Thus, we propose the use of a visual tool such as flow chart.

Table 3. Representation of the interaction flow between elements

\section{Representation of the interaction flow}

[Heart] [sends] if [Blood \{poor state\}] into [Lungs] else into [Organs].

[Heart] [Receive] [Blood \{riche state $\}$ from [Lungs] and [blood $\{$ poor state $\}]$ from [Organs].

Representation of the interaction flow with respecting the nature

[Physical Object 1] [sends] if [Physical Object 4 \{poor state\}] into [Physical Object 2] else into [Physical Object 3].

[Physical Object 1] [Receive] [Physical Object 4 \{riche state\}] from [Physical Object 2] and [Physical Object 4 \{riche state \}] from [Physical Object 2].

Representation of the interaction flow without respecting the nature

[Object 1] [sends] if [Object 4 \{poor state\}] into [Object 2] else into [Object 3].

[Object 1] [Receive] [Object 4 \{riche state\}] from [Object 2] and [Object 4 \{riche state\}] from [Object 2].

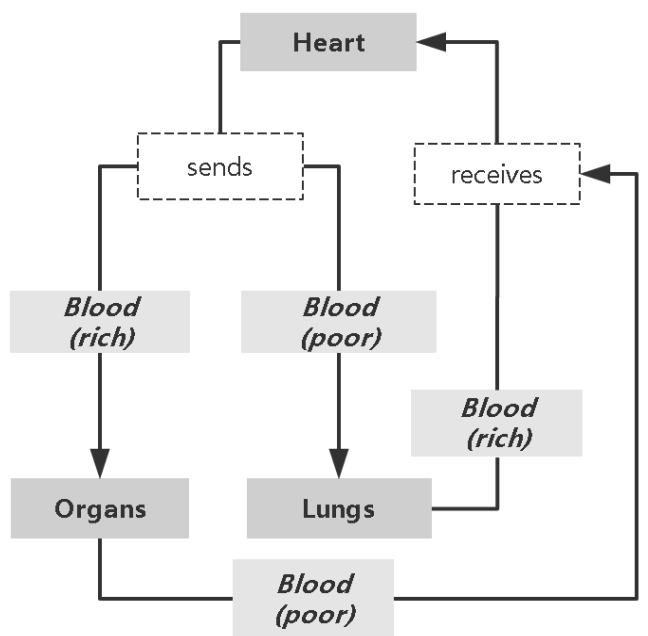

(a)

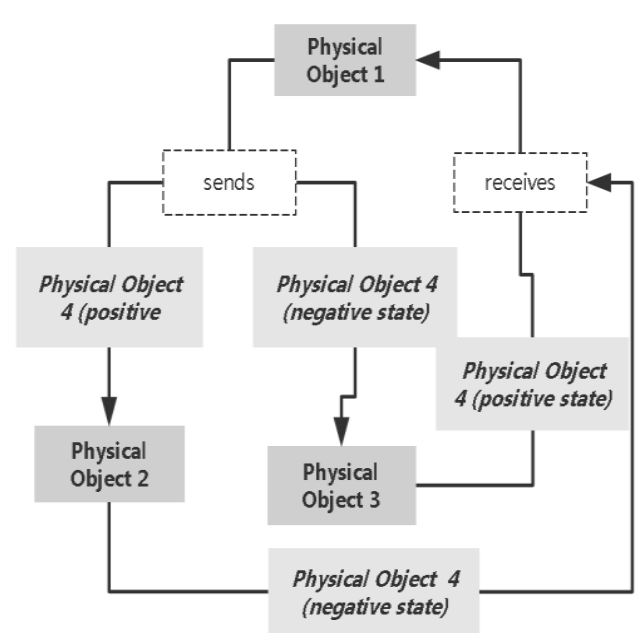

(b)

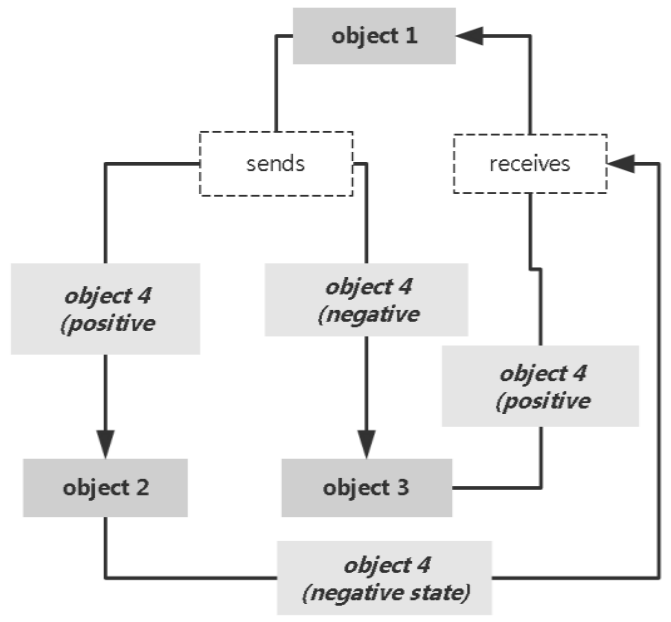

(c)

Fig.3. (a) Representation of the CS, (b) representation of CS respecting the nature of the elements, (c) representation of CS without respecting the nature of the elements.

Fig. 3 (a), represents the interaction flow of the CS. Fig. 3 (b) shows the interaction flow of elements respecting the nature of the elements of the CS, while Fig. 3 (c) shows the interaction flow without respecting their nature.

\section{Search of analogies for $C S$}

The representation of the interactions flows in Fig. 3 (b) and Fig. 3 (c) will be used in the search of analog examples. Fig. 4 shows a representation of WT that is analogous to the CS Fig. 2 (a).

From this perspective, the representation in Fig. 4 is an instance of the representation in Fig. 3 (c) and not of the one in Fig. 3 (b), since the element "Humans" is of a different nature than the element "Organs".

Therefore, the example in Fig. 3 (a) (source) and Fig. 4 (target) are analogs compared to the representation in Fig. 3 (c). 


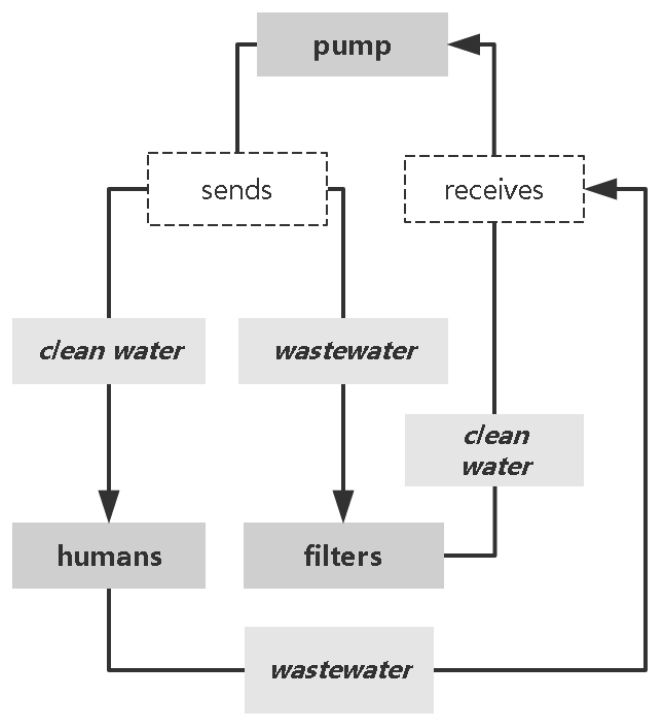

Fig.4. Analogue representation of the Wastewater treatment

The elements that constitute the WT are similar in nature to the CS elements, except for the element "humans" which is a "physical agent". This element can be replaced by another element that respects the nature of the source element which is a "physical object" such as a "House" or a "Nuclear Plant". This latter also involves replacing the "filter" element by a "cooler", the "clean water" by "cold water" and "waste water" by "hot water".

When the analog example does not contain all the elements of the field of study, the combination of several analogies from different fields becomes necessary. For example, the elements that make up a Power Plant and its relation with the consumers are similar to the Circulatory System, with the exception of the "cycle" concept, which can be represented by the "hydrological cycle".

\section{Learning and Assessment Strategies}

Respectively, the elements of the field of study, the analogs that do and do not respect the nature of the elements are organized in three levels called 3KL, which are: Domain Knowledge (DKL), Nature Domain Knowledge (NDKL) and Concept Domain Knowledge (CDKL).

The transition's order between the 3KL levels allows proposing learning and assessment strategies: Learning with analogies from the two levels NDKL and CDKL which uses simple representations either from other fields or real life, may help learners to better understand the field of study. Evaluating learners in the NDKL and CDKL levels helps to determine whether they are able to identify the elements of the field of study.

\section{THE SYSTEM DESIGN}

This section presents a system design that aims to integrate of analogies in the learning process. This system offers to the instructor the possibility to structure the desired knowledge and its analogies as activities. It also allows the definition of the progression, to test and get the feedback of several learning and assessment strategies. The system is expected to be integrated into existing Digital Learning Environment as a "plug-and-play" or as an independent learning support tool.

An overview of the system is shown in Fig. 5. It consists of the following blocks: in the Structured Knowledge block, the instructor defines the elements of the field of study and their analogies in the learning and the assessment stages respectively in the DKL, NDKL and CDKL levels.

The Strategy Block contains the strategies (defined by the instructor) to be tested during the learning process. The Activity Block contains the sequence of generated activities based on the Structured Knowledge and Strategy Blocks. The Result Interaction Block contains the results of the learner's interactions with the activities.

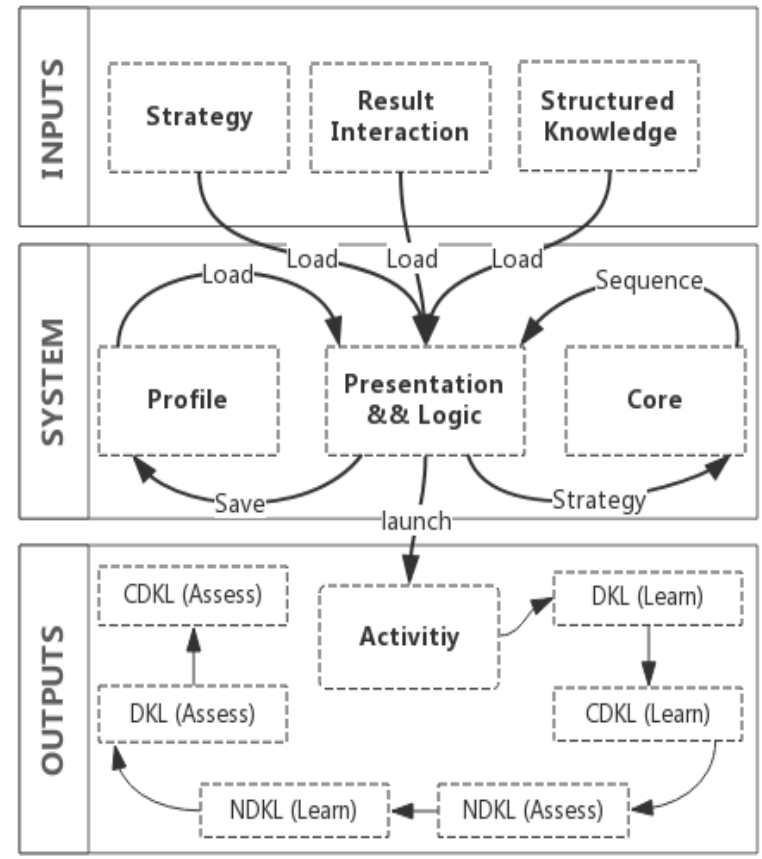

Fig.5. An overview of the proposed system.

During the learning process, the learner can be in a "learning" or "assessment" state. The integration of learning and assessment analogies allows us to introduce other states in addition to the states of the learning process. As presented in the previous section, the elements in the field of study and their analogies are organized in the $3 \mathrm{KL}$ levels.

Therefore, the learner may be in one of the following states: learning or assessment state in the DKL, in the NDKL or in the CDKL. The combination of these states in a specific order allows the development of several strategies.

In order to facilitate the encoding of these strategies by the core system, the use of a finite state automaton (Fig. 6) is adequate. It allows us to represent a given strategy by a sequence of numbers and characters, respecting the alphabet of the automaton. For example, the strategies '01ae2ae3ae45' and '01a2e45' designate respectively the activities in the following order: 
- Learn (DKL), Assess (DKL), Learn (NDKL), Assess (NDKL), Learn (CDKL), and Assess (CDKL).

- Learn (DKL) and Assess (NDKL).

The number in the start and in the end of the strategy are specific to the proposed system ' $0 * * * * * * * * * 45$ '.

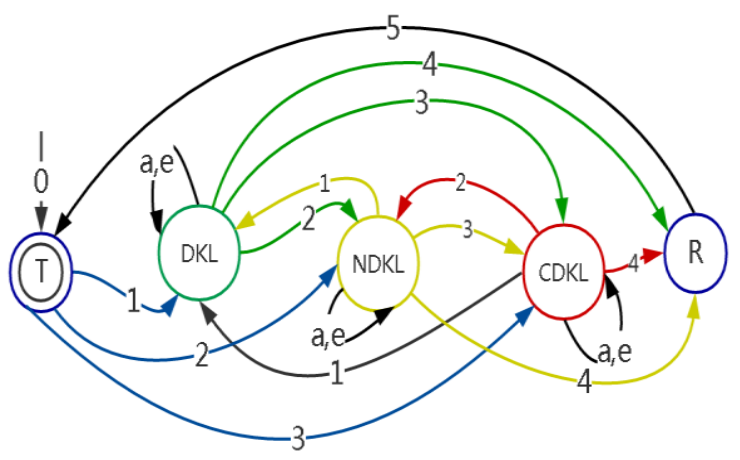

Fig.6. Design of the system core as by an automaton.

Fig. 7 represents the entities of the proposed system represented by a class diagram. The Hierarchy entity represents the structure of the desired knowledge, such as a summary and the self-relation represent the depth.

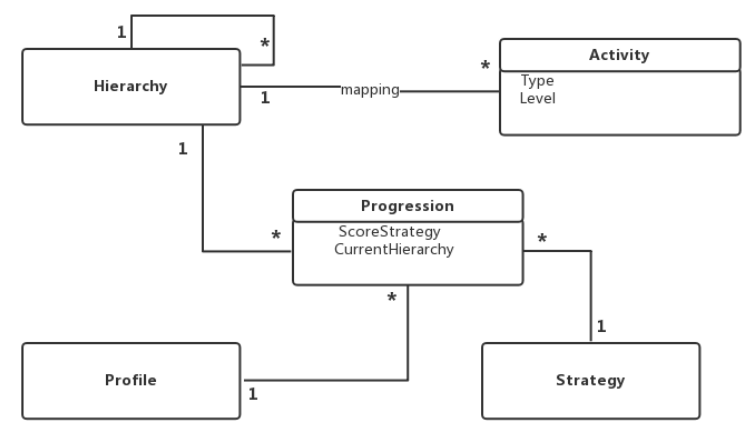

Fig.7. An entities class diagram of the proposed system

The Activity entity represents learning or an assessment activity specified by the attribute "type", the attributes "level" refers to the membership of the activity to one of the proposed 3KL levels. The Strategy entity represents strategies to be tested by the instructor. The Progression entity represents learner's progressions, strategies, and scores. The Profile entity contains learner's information.

Fig. 8 represents the interaction of a learner with the proposed system by a sequence diagram. After the authentication of the learner, the Presentation and Logic loads the current progression and the strategy to apply. This latter is then interpreted by the Core which returns a sequence of activities to the Presentation and Logic block which is responsible for the Human Machine Interface interaction (HMI) and the storage and retrieval of information and activities.

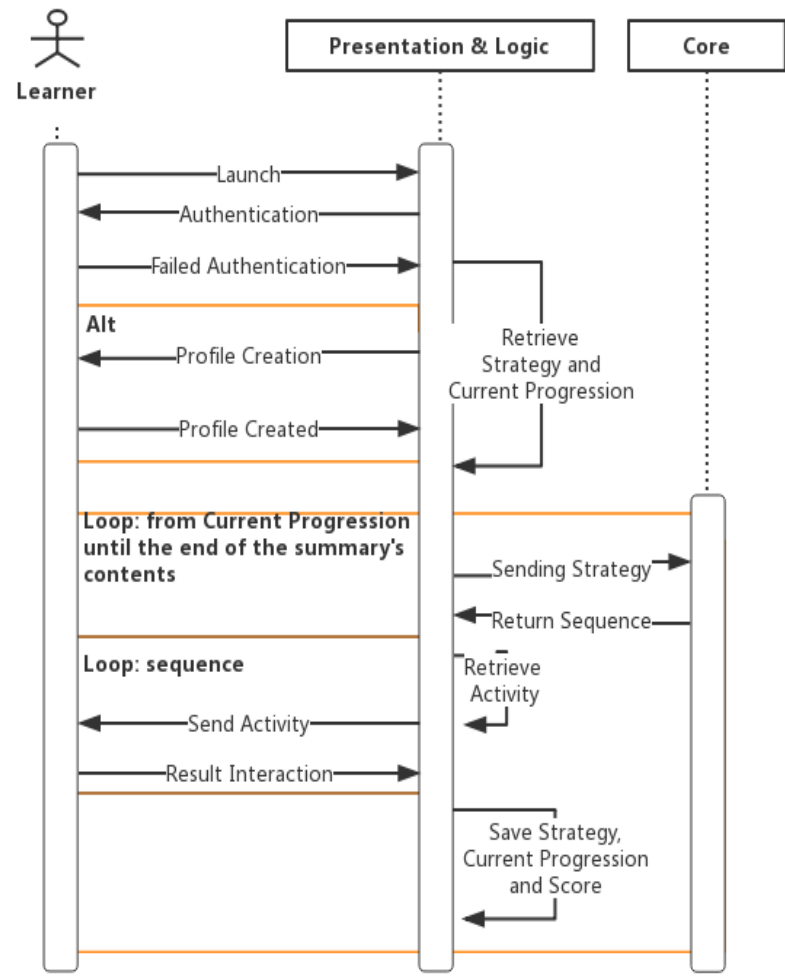

Fig.8. The design of interaction between the learner and the proposed system by a sequence diagram

\section{CONCLUSION}

This paper deals with the use of analogies in the learning process and its advantages. We have proposed a model and a process that support the design of analogies from static and dynamic aspects. To illustrate this, a case study is provided in which the different phases of the process are explained. These analogies are organized in what we called $3 \mathrm{KL}$ levels which allow the elaboration of several learning and assessment strategies. We have also proposed a design of the computer system that integrates the analogies in the learning process. This proposed system can be a "plug-and-play" or an independent learning support tool.

It's a given that the use of analogies in the learning process adds diversified activities that allow breaking the monotony of learning in a field of study. In addition, each learner has a different way of learning. Thus if we manage to find the adequate strategy for a given learner, he or she may have a better chance in learning and understanding any field of study.

The issue with the proposed model is its dependence on the skills of the instructors for structuring knowledge in the $3 \mathrm{KL}$ levels. Also, it still requires the definition of an abstract and limited vocabulary to describe the functions and the relations of any elements, allowing us to represent any field of study at a higher abstraction level with a unified language. 


\section{REFERENCES}

[1] Duit, R. (1991). On the role of analogies and metaphors in learning science. Science education, 75(6), 649-672.

[2] Zhang, F. (2014). L'application de l'analogie dans l'enseignement du français en Chine (Doctoral dissertation, Dijon).

[3] Gentner, D., Levine, S. C., Ping, R., Isaia, A., Dhillon, S., Bradley, C., \& Honke, G. (2016). Rapid learning in a children's museum via analogical comparison. Cognitive science, 40(1), 224-240.

[4] Gentner, D. (1983). Structure mapping: A theoretical framework for analogy. Cognitive science, 7(2), 155-170.

[5] Gregan-Paxton, J., \& John, D. R. (1997). Consumer learning by analogy: A model of internal knowledge transfer. Journal of Consumer Research, 24(3), 266-284.

[6] Gunning, T. G. (1996). Creating reading instruction for all children. Allyn and Bacon, Order Processing, PO Box 11071, Des Moines, IA 50336-1071.

[7] Harrison, A. G., \& Treagust, D. F. (1993). Teaching with analogies: A case study in grade 10 optics. Journal of Research in Science Teaching, 30(10), 1291-1307.

[8] Harrison, A. G., \& Treagust, D. F. (2006). Teaching and learning with analogies. In Metaphor and analogy in science education (pp. 11-24). Springer Netherlands.

[9] Holyoak, K. J., \& Thagard, P. (1989). Analogical mapping by constraint satisfaction. Cognitive science, 13(3), 295-355.

[10] Mayer, R. E. (2002). Rote versus meaningful learning. Theory into practice, 41(4), 226-232.

[11] Mestadi, W., Nafil, K., \& Touahni, R. (2015, October). Knowledge structuring for learning by level. In Intelligent Systems: Theories and Applications (SITA), 2015 10th International Conference on (pp. 1-6). IEEE.

[12] Moreno, R., Ozogul, G., \& Reisslein, M. (2011). Teaching with concrete and abstract visual representations: Effects on students' problem solving, problem representations, and learning perceptions. Journal of Educational Psychology, 103(1), 32.

[13] Sander, E. (2002). L'Analogie, fondement de nos apprentissages. La Recherche, (353), 40-43.

[14] Sloutsky, V. M., Kaminski, J. A., \& Heckler, A. F. (2005). The advantage of simple symbols for learning and transfer. Psychonomic bulletin \& review, 12(3), 508-513.

[15] Tardif, J., \& Meirieu, P. (1996). Stratégie pour favoriser le transfert des connaissances. Vie pédagogique, 98(7), 4-7.

[16] Williams, D., Ma, Y., Feist, S., Richard, C. E., \& Prejean, L. (2007). The design of an analogical encoding tool for game-based virtual learning environments. British Journal of Educational Technology, 38(3), 429-437.

[17] Winston, P. H. (1980). Learning and reasoning by analogy. Communications of the ACM, 23(12), 689-703.

[18] Qin, G., Sun, S., Ye, H., \& Lu, D. (2011). Strategies on Teaching Reformation for Mechanical Manufacturing Technology. International Journal of Education and Management Engineering (IJEME), 1(6), 70.

\section{Authors' Profiles}

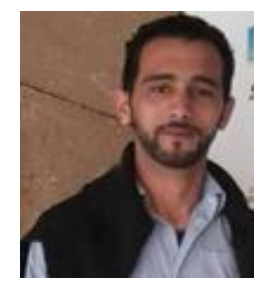

Mestadi Walid received his M.S. degree in software quality in 2012 from Ibn Tofail University, Faculty of science, Kenitra- Morocco. Currently he is preparing a $\mathrm{PhD}$ at the same faculty. His research interests include learning, serious games, software architecture and artificial intelligence.

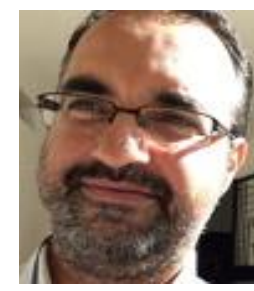

Khalid Nafil is currently professor of Software Engineering at University Mohammed V in Rabat - ENSIAS. He received his $\mathrm{PhD}$ in Computer Science from the University Mohammed $\mathrm{V}$ in Rabat, Morocco since 1997. His main research interests are Cost Estimation, Software Testing, Serious Games, Software Process Improvement, Software Engineering Education.

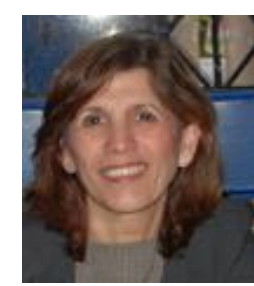

Touahni Raja is currently professor at Ibn Tofail University, Faculty of science, Kenitra- Morocco. She received his $\mathrm{PhD}$ in Microelectronics from the University Paul Sabatier, Toulouse, France, in September 1986. In 2001, she received the Ph.D degree in the field of Data Analysis at the same faculty. His main research interests are Image Processing, Data Analysis, Artificial Intelligence, Cognitive Science and Artificial Thinking, Computer Vision.

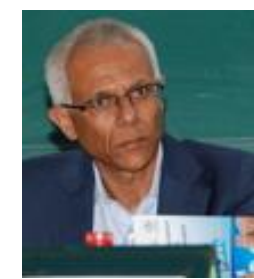

Messoussi Rochdi is currently professor at Ibn Tofail University, Faculty of science, Kenitra- Morocco. He received his $\mathrm{PhD}$ degree in solid state physics from the University of Nantes, France, in 1990. In 2001 he received the master degree in the use of IT for learning from the University of Strasbourg, France. For the last 15 years, he has been carrying out research, about Human Computer Interaction to promote learning and education. His main research interests are Artificial Intelligence, Computing in Social science, Arts and Humanities. 
How to cite this paper: Walid Mestadi, Khalid Nafil, Raja Touahni, Rochdi Messoussi,"Knowledge Representation by Analogy for the Design of Learning and Assessment Strategies", International Journal of Modern Education and Computer Science(IJMECS), Vol.9, No.6, pp.9-16, 2017.DOI: 10.5815/ijmecs.2017.06.02 